\title{
COMPLETELY POSITIVE MAPS ON COXETER GROUPS AND THE ULTRACONTRACTIVITY OF THE $q$-ORNSTEIN-UHLENBECK SEMIGROUP
}

\author{
MAREK BOŻEJKO \\ Institute of Mathematics, University of Wroctaw \\ pl. Grunwaldzki 2/4, 50-384 Wroctaw, Poland \\ E-mail: bozejko@math.uni.wroc.pl
}

1. Coxeter groups. In this note we give an application of the following result on the symmetric group $S_{n}$ :

THEOREM 1. For fixed $n \in \mathbf{N}$ let us consider the permutation group $S_{n}$ and denote by $\pi_{i} \in S_{n}(i=1, \ldots, n-1)$ the transposition between $i$ and $i+1$. Furthermore, let operators $T_{i} \in B(\mathcal{H})(i=1, \ldots n-1)$ on some Hilbert space $\mathcal{H}$ be given, with the properties:

(i) $T_{i}^{*}=T_{i}$ for all $i=1, \ldots, n-1$;

(ii) $\left\|T_{i}\right\| \leq 1$ for all $i=1, \ldots, n-1$;

(iii) The $T_{i}$ satisfy the braid relations:

$$
\begin{gathered}
T_{i} T_{i+1} T_{i}=T_{i+1} T_{i} T_{i+1} \text { for all } i=1, \ldots, n-2, \\
T_{i} T_{j}=T_{j} T_{i} \text { for all } i, j=1, \ldots, n-1 \text { with }|i-j| \geq 2 .
\end{gathered}
$$

Now let us define a function

$$
\varphi: S_{n} \longrightarrow B(\mathcal{H})
$$

by quasi-multiplicative extension of

$$
\varphi(e)=1, \quad \varphi\left(\pi_{i}\right)=T_{i},
$$

i.e. for a reduced word $S_{n} \ni \sigma=\pi_{i(1)} \ldots \pi_{i(k)}$ we put $\varphi(\sigma)=T_{i(1)} \ldots T_{i(k)}$. Then $\varphi$ is a completely positive map, i.e. for all $l \in \mathbf{N}, f_{i} \in \mathbf{C} S_{n}, x_{i} \in \mathcal{H}(i=1, \ldots, l)$ we have

$$
\left\langle\sum_{i, j=1}^{l} \varphi\left(f_{j}^{*} f_{i}\right) x_{i}, x_{j}\right\rangle \geq 0 .
$$

1991 Mathematics Subject Classification: Primary 51F15, $20 \mathrm{~F} 55$.

Research partially supported by KBN grant 2P03A05108.

The paper is in final form and no version of it will be published elsewhere. 
By our previous result from [BSp1], Theorem 1 is equivalent to the following:

THEOREM 2. Under the assumptions of Theorem 1 the operator

$$
\begin{aligned}
P^{(n)} & =P_{T}^{(n)}=\sum_{\sigma \in S_{n}} \varphi(\sigma)= \\
& =\left(1+T_{1}\right)\left(1+T_{2}+T_{2} T_{1}\right) \ldots\left(1+T_{n-1}+T_{n-1} T_{n-2}+\cdots+T_{n-1} \ldots T_{1}\right)
\end{aligned}
$$

satisfies

$$
P^{(n)} \geq \prod_{k=2}^{n} c_{k}(q)>0
$$

where

$$
c_{k}(q)=\left(1-q^{2}\right)^{-1} \prod_{l=1}^{k}\left(1-q^{l}\right)\left(1+q^{l}\right)^{-1} .
$$

Moreover, by Gauss formula

$$
c_{k}(q) \geq c(q)=(1-q)^{-1} \prod_{l=1}^{\infty}\left(1-q^{l}\right)\left(1+q^{l}\right)^{-1}=(1-q)^{-1} \sum_{l=-\infty}^{+\infty}(-1)^{l} q^{l^{2}} .
$$

In the proof we need the following lemma:

Lemma 3. If $T_{i} \in B(\mathcal{H})$ satisfy the braid relations of Theorem 1 , then for $1 \leq r<$ $k<n-1$, we have

$$
\left(T_{n-1} T_{n-2} \ldots T_{k}\right)\left(T_{n-1} T_{n-2} \ldots T_{r}\right)=T_{n-1}\left(T_{n-1} T_{n-2} \ldots T_{r}\right)\left(T_{n-1} \ldots T_{k+1}\right) .
$$

Proof. The proof of the Lemma follows by induction on $k$ :

Let $k=n-2$. Then by the braid relations we get

$$
\begin{aligned}
& (T_{n-1} \underbrace{\left.T_{n-2}\right)\left(T_{n-1} T_{n-2}\right.} T_{n-3} \ldots T_{r})= \\
& =T_{n-1} \underbrace{T_{n-1} T_{n-2} T_{n-1}} T_{n-3} \ldots T_{r}= \\
& =T_{n-1}\left(T_{n-1} T_{n-2} T_{n-3} \ldots T_{r}\right) T_{n-1} .
\end{aligned}
$$

The next step looks as follows:

$$
\begin{aligned}
& \left(T_{n-1} T_{n-2} T_{n-3}\right)\left(T_{n-1} T_{n-2} T_{n-3} \ldots T_{r}\right)= \\
& =\left(T_{n-1} T_{n-2}\right)(T_{n-1} \underbrace{T_{n-3} T_{n-2} T_{n-3}} \ldots T_{r})= \\
& =(T_{n-1} \underbrace{\left.T_{n-2}\right)\left(T_{n-1} T_{n-2}\right.} T_{n-3} T_{n-2} T_{n-4} \ldots T_{r})= \\
& =T_{n-1} T_{n-1} T_{n-2} T_{n-1} T_{n-3} T_{n-2}\left(T_{n-4} \ldots T_{r}\right)= \\
& =T_{n-1} T_{n-1} T_{n-2} T_{n-3}\left(T_{n-1} T_{n-2}\right)\left(T_{n-4} \ldots T_{r}\right)= \\
& =T_{n-1}\left(T_{n-1} T_{n-2} T_{n-3} \ldots T_{r}\right)\left(T_{n-1} T_{n-2}\right) .
\end{aligned}
$$

Next we need the following important lemma:

LEMma 4. Let $T_{i} \in B(\mathcal{H})$ and

$$
R_{k}\left(T_{1}, \ldots, T_{k-1}\right)=R_{k}=1+T_{k-1}+T_{k-1} T_{k-2}+\ldots+T_{k-1} T_{k-2} \ldots T_{1},
$$

where $k=2,3, \ldots, n$. Then 
(a) $\quad R_{k}\left(1-T_{k-1} T_{k-2} \ldots T_{1}\right)=$

$=\left(1-T_{k-1}^{2} T_{k-2} \ldots T_{1}\right)\left(1+T_{k-1}+T_{k-1} T_{k-2}+\ldots+T_{k-1} T_{k-2} \ldots T_{2}\right)=$

$=\left(1-T_{k-1}^{2} T_{k-2} \ldots T_{1}\right) R_{k-1}\left(T_{2}, T_{3}, \ldots, T_{k-1}\right)$,

(b) $\quad R_{n}\left(1-T_{n-1} T_{n-2} \ldots T_{2} T_{1}\right)\left(1-T_{n-1} T_{n-2} \ldots T_{2}\right) \ldots\left(1-T_{n-1}\right)=$

$=\left(1-T_{n-1}^{2} T_{n-2} \ldots T_{2} T_{1}\right)\left(1-T_{n-1}^{2} T_{n-2} \ldots T_{2}\right) \ldots\left(1-T_{n-1}^{2} T_{n-2}\right)\left(1+T_{n-1}\right)$.

Pro of. Let us start with the case $k=3$. Since $R_{3}=1+T_{2}+T_{2} T_{1}$, we have

$$
\begin{aligned}
R_{3}\left(1-T_{2} T_{1}\right) & =1+T_{2}-T_{2}^{2} T_{1}-T_{2} T_{1} T_{2} T_{1}= \\
& =1+T_{2}-T_{2}^{2} T_{1}-T_{2}^{2} T_{1} T_{2}= \\
& =\left(1-T_{2}^{2} T_{1}\right)\left(1+T_{2}\right) .
\end{aligned}
$$

Now we consider the case $k=4$. By natural calculations using Lemma 3 we get

$$
\begin{aligned}
R_{4}\left(1-T_{3} T_{2} T_{1}\right) & =\left(1+T_{3}+T_{3} T_{2}\right)-\left(T_{3}^{2} T_{2} T_{1}\right)\left(1+T_{3}+T_{3} T_{2}\right)= \\
& =\left(1-T_{3}^{2} T_{2} T_{1}\right)\left(1+T_{3}+T_{3} T_{2}\right) .
\end{aligned}
$$

Therefore, using the case $k=3$, we have

$$
R_{4}\left(1-T_{3} T_{2} T_{1}\right)\left(1-T_{3} T_{2}\right)=\left(1-T_{3}^{2} T_{2} T_{1}\right)\left(1-T_{3}^{2} T_{2}\right)\left(1+T_{2}\right) .
$$

Repeating this process we get the proof of the Lemma.

This implies the next lemma.

LEMMA 5. If

$$
\begin{aligned}
P^{(n)} & =\sum_{\sigma \in S_{n}} \varphi_{T}(\sigma)=P^{(n-1)}\left(1+T_{n-1}+\ldots+T_{n-1} \ldots T_{1}\right)= \\
& =P^{(n-1)} R_{n}=R_{2} R_{3} \ldots R_{n},
\end{aligned}
$$

and $\left\|T_{i}\right\| \leq q<1$, then

$$
\left\|R_{n}^{-1}\right\| \leq(1-q)^{-1} \prod_{k=1}^{n-1}\left(1+q^{k}\right) \prod_{k=3}^{n}\left(1-q^{k}\right)^{-1} .
$$

Pr o of. By Lemma 4 we have

$$
R_{n}=\prod_{k=1}^{n-2}\left(1-T_{n-1}^{2} T_{n-2} \ldots T_{k}\right)\left(1+T_{n-1}\right) \prod_{l=n-1}^{1}\left(1-T_{n-1} \ldots T_{l}\right)^{-1} .
$$

But, since $\left\|T_{i}\right\|<q<1$, therefore

$$
\left\|\left(1-T_{n-1} \ldots T_{(n-1)-k}\right)^{-1}\right\| \leq\left(1-q^{k}\right)^{-1}
$$

and we infer the estimation of Lemma 5 .

Now we can state Theorem 2 in a stronger version.

THEOREM 6. If $\left\|T_{i}\right\| \leq q<1$ and the assumptions of Theorem 1 are satisfied, then (i)

$$
P^{(n)} \geq \omega(q)\left(P^{(n-1)} \otimes 1\right),
$$


where

$$
\begin{gathered}
\omega(q)^{2}=\left(1-q^{2}\right)^{-1} \prod_{k=1}^{\infty}\left(1-q^{k}\right)\left(1+q^{k}\right)^{-1} . \\
P^{(n)} \leq \frac{1}{1-q}\left(P^{(n-1)} \otimes 1\right) .
\end{gathered}
$$

Proof. The proof follows from the following considerations:

(a) We know from the results of [BSp1] that

$$
P^{(n)} \geq 0 \text {. }
$$

Since, by Lemma $5,\left\|R_{n}^{-1}\right\| \leq \frac{1}{c}$ for some $c>0$, therefore

and this implies

$$
\left\|\left(R_{n}^{-1}\right)^{*} R_{n}^{-1}\right\| \leq \frac{1}{c^{2}}
$$

$$
R_{n} R_{n}^{*} \geq c^{2}
$$

But, because

$$
P^{(n)}=\left(P^{(n-1)} \otimes 1\right) R_{n},
$$

and $P^{(n)}=P^{(n) *}$, we obtain

$$
\left[P^{(n)}\right]^{2}=P^{(n-1)} R_{n} R_{n}^{*} P^{(n-1)} \geq c^{2}\left[P^{(n-1)}\right]^{2}
$$

and hence

$$
P^{(n)} \geq c\left(P^{(n-1)} \otimes 1\right) \text {, where } c=\omega(q) .
$$

(b) The statement (ii) of Theorem 2 follows from the two facts:

$$
P^{(n)}=P^{(n-1)} R_{n}
$$

and

$$
R_{n}=1+T_{n-1}+T_{n-1} T_{n-2}+\ldots+T_{n-1} \ldots T_{1} .
$$

Therefore $\left\|R_{n}\right\|<\frac{1}{1-q}$ and again as before we have

$$
P^{(n)} \geq \frac{1}{1-q}\left(P^{(n-1)} \otimes 1\right) .
$$

So, the proof of Theorem 6 is complete.

This theorem is also valid for all finite and affine Coxeter groups (for more details see [BSp4]). Theorem 1 comes from investigations in harmonic analysis on groups (see [B1], [BSz]) and on perturbed cannonical commutation relations. In the paper with $\mathrm{R}$. Speicher ([BSp1]) we considered the following relations

$$
c_{i} c_{j}^{*}-q c_{j}^{*} c_{i}=\delta_{i j} \mathbf{1}
$$

for a real $q$ with $|q| \leq 1$, and we needed essentially the fact that the function

$$
\varphi: S_{n} \longrightarrow \mathbf{C}, \quad \pi \longmapsto q^{|\pi|}
$$

is a positive definite function for all $n$, where $|\pi|$ denotes the number of inversions of $\pi$. For other proofs of that result see [BKS, BSp1, BSp2, BSp4, BSz, Spe, Z]. 
R. Speicher in [Spe] considered more general commutations relations

for

$$
d_{i} d_{j}^{*}-q_{i j} d_{j}^{*} d_{i}=\delta_{i j} \mathbf{1}
$$

$$
-1 \leq q_{i j}=q_{j i} \leq 1,
$$

and he founded the existence of a Fock representation by central limit arguments. Our construction of the $q_{i j}$ relations depends on some operator $\mathrm{T}$ which is a self-adjoint contraction on a Hilbert space $\mathcal{H}$ and satisfies the braid or Yang-Baxter relations of the following form:

$$
T_{1} T_{2} T_{1}=T_{2} T_{1} T_{2},
$$

where $T_{1}=T \otimes \mathbf{1}$ and $T_{2}=\mathbf{1} \otimes T$ on $\mathcal{H} \otimes \mathcal{H} \otimes \mathcal{H}$ are the natural amplifications of $\mathrm{T}$ to $\mathcal{H} \otimes \mathcal{H} \otimes \mathcal{H}$

From Theorem 1 we get more general construction of deformed commutation relations of the Wick form:

$$
d_{i} d_{j}^{*}-\sum_{r, s} t_{j s}^{i r} d_{r}^{*} d_{s}=\delta_{i j} \mathbf{1}
$$

(see also Jorgensen et al. [JSW] and [BSp4] for similar considerations).

2. Applications. Next we examine the deformed commutation relations from an operator spaces' point of view. If we assume that $\|T\|=q \leq 1$ and if we take $G_{i}=d_{i}+d_{i}^{*}$, then we prove that the operator space generated by the $G_{i}$ is completely isomorphic to the canonical operator Hilbert space $\mathcal{R} \cap \mathcal{C}$, which means

$$
\left\|\sum_{i=1}^{N} a_{i} \otimes G_{i}\right\| \approx \max \left(\left\|\sum_{i=1}^{N} a_{i} a_{i}^{*}\right\|^{1 / 2},\left\|\sum_{i=1}^{N} a_{i}^{*} a_{i}\right\|^{1 / 2}\right)
$$

for all bounded operators $a_{1}, \ldots, a_{N}$ on some Hilbert space. This generalizes the Theorem of Haagerup and Pisier [HP], who obtained that result for free creation and annihilation operators, (see also [VDN] and [Buch]). As another application of our construction we have obtained a large class of non-injective von Neumann algebras, when considering the von Neumann algebra $V N\left(G_{1}, \ldots, G_{N}\right)$ generated by $G_{1}, \ldots, G_{N}$. For more details see [BSp4, BKS].

3. The ultracontractivity of the $q$-second quantization functor $\Gamma_{q}$. Let $T$ : $\mathcal{H} \longrightarrow \mathcal{K}$ be a contraction beetween real Hilbert spaces. Then the linear map defined on elementary tensors by

$$
F_{q}(T)\left(f_{1} \otimes \ldots \otimes f_{n}\right)=T f_{1} \otimes \ldots \otimes T f_{n}
$$

extends to a contraction from $q$-Fock spaces $F_{q}(\mathcal{H})$ to $F_{q}(\mathcal{K})$. Here $F_{q}(\mathcal{H})$ is the completion of the full Fock space $\bigoplus_{n=0}^{\infty} \mathcal{H}^{\otimes n}$ with respect to the new scalar product

$$
\left\langle f_{1} \otimes \ldots \otimes f_{n}, g_{1} \otimes \ldots \otimes g_{n}\right\rangle_{q}=\delta_{n, m} \sum_{\sigma \in S_{n}} q^{i n v(\sigma)}\left\langle f_{\sigma(1)}, g_{1}\right\rangle \ldots\left\langle f_{\sigma(n)}, g_{n}\right\rangle .
$$

The creation operators are defined as:

$$
c^{*}\left(f_{0}\right)\left(f_{1} \otimes \ldots \otimes f_{n}\right)=f_{0} \otimes f_{1} \otimes \ldots \otimes f_{n}, \quad f_{j} \in \mathcal{H}
$$

and $c(f)=\left[c^{*}(f)\right]^{*}$. 
Let $G(f)=c(f)+c^{*}(f)$ for $f \in \mathcal{H}$. Let $\Gamma_{q}(\mathcal{H})$ be the von Neumann algebra generated by $G(f), f \in \mathcal{H}$, and

$$
\tau_{q}(S)=\langle S \Omega, \Omega\rangle_{q}, \quad S \in \Gamma_{q}(\mathcal{H})
$$

One can show that $\tau_{q}$ is a trace on $\Gamma_{q}(\mathcal{H})$.

If $\operatorname{dim} \mathcal{H}=\infty$, then we showed that $\Gamma_{q}(\mathcal{H})$ is a factor.

If $e_{1}, e_{2}, \ldots, e_{N}$ is an orthonormal basis of $\mathcal{H}$, then we put $G_{i}=G\left(e_{i}\right),(i=1, \ldots, N$, $N=\infty, 1,2, \ldots)$. In this setting the following theorem holds:

TheOREm 7 ([BKS], Theorem 2.1.1). Let $T$ be as above, then there exists a unique map $\Gamma_{q}(T): \Gamma_{q}(\mathcal{H}) \longrightarrow \Gamma_{q}(\mathcal{K})$ such that $\Gamma_{q}(T)(X) \Omega=F_{q}(T)(X \Omega)$ for every $X \in \Gamma_{q}(\mathcal{H})$. The map $\Gamma_{q}(T)$ is bounded, normal, unital, completely positive and trace preserving.

We note that $\Gamma_{q}$ is a functor, namely if $S: \mathcal{H} \longrightarrow \mathcal{K}$ and $T: \mathcal{K} \longrightarrow \mathcal{J}$ are contractions, then $\Gamma_{q}(S T)=\Gamma_{q}(S) \Gamma_{q}(T)$.

If $\mathcal{H}$ is a real Hilbert space and $T_{t}=e^{-t} I$ for $t \geq 0$, then the completely positive maps $P_{t}^{q}=\Gamma_{q}\left(T_{t}\right), t \geq 0$, on $\Gamma_{q}(\mathcal{H})$, form a semigroup, called the $q$-Ornstein-Uhlenbeck semigroup. The $q$-Ornstein-Uhlenbeck semigroup extends to a semigroup of contractions of the non-commutative $L^{p}$ spaces, which are symmetric on $L^{2}$. Its infinitesimal generator on $L^{2}$ is the number operator $N^{q}$, i.e. $P_{t}=\exp \left(-t N^{q}\right)$, where $N^{q}$ is the unbounded self-adjoint operator defined as $N^{q} \Omega=0$ and

$$
N^{q} f_{1} \otimes \ldots \otimes f_{n}=n f_{1} \otimes \ldots \otimes f_{n}, \quad f_{1}, \ldots, f_{n} \in \mathcal{H} .
$$

$\mathrm{Ph}$. Biane [Bia] proved Nelson's hypercontractivity of the $q$-Ornstein-Uhlenbeck semigroup $P_{t}$, extending the results of Nelson and Gross. In that paper Ph. Biane also showed ultracontractivity for $q=0$ using some results of the author (see [B2]). Now we prove the ultracontractivity of that semigroup for all $q \in[-1,1]$.

Theorem 8. Let $X$ be in the eigenspace of $N^{q}$, with eigenvalue $n$. Then

(i) $\|X\|_{L^{\infty}} \leq C(q)(n+1)\|X\|_{L}^{2}$;

(ii) For $t \geq 0, P_{t}$ maps $L^{2}$ into $L^{\infty}=V N_{q}\left(G_{1} \ldots G_{N}\right)$ and for $t \leq 1$

$$
\left\|P_{t}^{q}\right\|_{L^{2} \rightarrow L^{\infty}} \leq c_{q} t^{-3 / 2}
$$

(iii) (Poincaré-Sobolev inequality). If $Q_{q}(X)=\left\langle X N^{q} X \Omega, \Omega\right\rangle$ is a non-commutative complete Dirichlet form (on an appropriate domain) on $L^{2}\left(\Gamma_{q}(\mathcal{H}), \tau_{q}\right)$, in the sense of $[D L]$, then there exists a constant $c_{q} \geq 0$ such that for all $X$ in the domain of $Q_{q}$ we have

$$
\|X\|_{L^{3}}^{2} \leq c_{q}\left(\left|\tau_{q}(X)\right|^{2}+Q_{q}(X)\right)
$$

For the details of the proof of this theorem see [B3] and [Bia].

\section{References}

[B] D. Bakry, L'hypercontractivité et son utilisation en théorie des semigroupes, Lectures on Probability Theory, Lecture Notes in Mathematics, vol.1581, Springer, Berlin Heidelberg New York, (1994), 1-114.

[Bia] Ph. Biane, Free Hypercontractivity, Prepublication N 350, Paris VI, (1996), 1-19. 
[B1] M. Bożejko, Positive definite kernels, length functions on groups and a noncommutative von Neumann inequality, Studia Math. 95 (1989), 107-118.

[B2] M. Bożejko, A q-deformed probability, Nelson's inequality and central limit theorems, Non-linear fields, classical, random, semiclassical (P. Garbaczewski and Z. Popowicz, eds.), World Scientific, Singapore, (1991), 312-335.

[B3] M. Bożejko, Ultracontractivity and strong Sobolev inequality for q-Ornstein-Uhlenbeck semigroup (to appear).

[BSp1] M. Bożejko and R. Speicher, An Example of a Generalized Brownian Motion, Comm. Math. Phys. 137 (1991), 519-531.

[BSp2] M. Bożejko and R. Speicher, An Example of a generalized Brownian motion II. In : Quantum Probability and Related Topics VII (ed. L. Accardi), Singapore: World Scientific (1992), 219-236.

[BSp3] M. Bożejko and R. Speicher, Interpolation between bosonic and fermionic relations given by generalized Brownian motions, Math. Zeit. 222 (1996), 135-160.

[BSp4] M. Bożejko and R. Speicher, Completely positive maps on Coxeter Groups, deformed commutation relations, and operator spaces, Math. Annalen 300 (1994), 97-120.

[BKS] M. Bożejko, B. Kümmerer and R. Speicher, q-Gaussian Processes: Non-commutative and Classical Aspects, Comm. Math. Phys. 185 (1997),129-154.

[BSz] M. Bożejko and R. Szwarc, Algebraic length and Poincare series on reflection groups with applications to representations theory, University of Wrocław, preprint (1996), $1-25$.

[Buch] A. Buchholz, Norm of convolution by operator-valued functions on free groups, Proc. Amer. Math. Soc., (1997).

[CL] E. A. Carlen and E. H. Lieb, Optimal hypercontractivity for Fermi fields and related non-commutative integration inequalities, Comm. Math. Phys. 155 (1993), 27-46.

[CSV] T. Coulhon, L. Saloff-Coste, and N. Th. Varopoulos, Analysis and Geometry on Groups, Cambgidge Tracts in Mathematics, vol.100, Cambridge University Press, 1992.

[DL] E. B. Davies and J. M. Lindsay, Non-commutative symmetric Markov semigroups, Math. Zeit. 210 (1992), 379-411.

[G] L. Gross, Hypercontractivity and logarithmic Sobolev inequalities for the CliffordDirichlet form, Duke Math. J. 42 (1975), 383-396.

[H] U. Haagerup, An example of a non nuclear $C^{*}$ - algebra which has the metric approximation property, Invent. Math. 50 (1979), 279-293.

[HP] U. Haagerup and G. Pisier, Bounded linear operators between $C^{*}$-algebras, Duke Math. J. 71 (1993), 889-925.

[JSW] P. E. T. Joergensen, L. M. Schmitt and R. F. Werner, Positive representations of general commutation relations allowing Wick ordering, J. Func. Anal., 134 (1995),3-99.

[N] E. Nelson, The free Markoff field, J. Func. Anal. 12 (1973), 211-227.

[P] G. Pisier, The Operator Hilbert Space OH, Complex Interpolation and Tensor Norms, Mem. Amer. Math.Soc. 1996.

[Spe] R. Speicher, A non commutative central limit theorem, Math. Zeit. 209 (1992), 55-66.

[VDN] D. V. Voiculescu, K. Dykema and A. Nica, Free random variables, CRM Monograph Series No.1, Amer. Math. Soc., Providence, RI, 1992.

[Z] D. Zagier, Realizability of a model in infinity statistics, Comm. Math. Phys. 147 (1992), 199-210. 